\title{
The first pediatric case of leukoencephalopathy with brainstem and spinal cord involvement and lactate elevation (LBSL) from Turkey
}

\author{
Dilek Çavuşoğlu, ${ }^{1}$ Nihal Olgaç-Dündar, ${ }^{1}$ Özgür Öztekin, ${ }^{2}$ Taha Reşid Özdemir, ${ }^{3}$ \\ Pınar Arıcan, ${ }^{4}$ Pinar Gençpinar ${ }^{1}$ \\ Department of ${ }^{1}$ Pediatric Neurology, Faculty of Medicine, Izmir Katip Celebi University; Departments of ${ }^{2}$ Radiology, \\ ${ }^{3}$ Medical Genetics, and ${ }^{4}$ Pediatric Neurology, Tepecik Training and Research Hospital, İzmir, Turkey \\ E-mail: nodundar@gmail.com \\ Received : 17th April 2017, Revised 25th May 2017, Accepted : 30th June 2017
}

SUMMARY: Çavuşoğlu D, Olgaç-Dündar N, Öztekin Ö, Özdemir TR, Arıcan P, Gençpınar P. The first pediatric case of leukoencephalopathy with brainstem and spinal cord involvement and lactate elevation (LBSL) from Turkey. Turk J Pediatr 2018; 60: 216-220.

Leukoencephalopathy with brainstem and spinal cord involvement and lactate elevation (LBSL) is defined as an autosomal recessive inheritance disorder characterized by slowly progressive cerebellar, pyramidal and dorsal column dysfunction. The diagnosis is based on specific magnetic resonance imaging abnormalities (MRI) in the cerebral and cerebellar white matter and selective involvement of white matter tracts in the brain stem and spinal cord. LBSL is caused by mutations in the DARS2 gene which encodes the mitochondrial aspartyl-tRNA synthetase. Herein, we report the first pediatric case from Turkey with a typical MRI course of LBSL associated with a compound heterozygous mutation in DARS2 gene.

Key words: LBSL, DARS2, leukoencephalopathy, mitochondrial, MRI.

Leukoencephalopathy with brainstem and spinal cord involvement and lactate elevation (LBSL) is a rare autosomal recessive disorder of white matter. ${ }^{1}$ LBSL is also a mitochondrial disease caused by homozygous or compound heterozygous mutation in DARS2 gene encoding mitochondrial aspartyl-tRNA synthetase (mtAspRS). It is characterized by slowly progressive cerebellar, pyramidal, and dorsal column dysfunction. ${ }^{2}$ The diagnosis is based on distinctive magnetic resonance imaging (MRI) abnormalities in the cerebral and cerebellar white matter and specific white matter tracts in the brain stem and spinal $\operatorname{cord}^{3}$. Lactate elevation in the abnormal white matter is an extensive, but not invariable finding in magnetic resonance spectroscopy. 2,4

To our knowledge, we describe the first pediatric patient from Turkey diagnosed with LBSL based on the clinical, molecular genetics and neuroradiological outcomes.

\section{Case Report}

A 12-year-old male patient was admitted to the pediatric neurology outpatient clinic with a complaint of headache located on bilateral frontal regions repeating twice a week for the past three years. The patient was born as the second child of unrelated parents, after an uneventful pregnancy and delivery at full term. His birth weight was $3400 \mathrm{~g}$. His developmental skills were normal. He required operations due to cataract of the right eye twice when he was seven months and seven years old. On admission, his neurological examination revealed a right dilated pupil not responsive to light with loss of visual acuity in the involved eye, hyperactive deep tendon reflexes, bilateral Babinski signs and moderate spasticity of legs. We could not determine cognitive dysfunction. Complete blood count, routine biochemistry, creatine phosphokinase, plasma lactate, tandem mass spectrometry, blood and 
urine quantitative amino acids, urine organic acids, very long chain fatty acids (VLCFAs), phytanic acid and pristanic acid levels were normal. Lysosomal enzymes (including arylsulfatase A and galactocerebrosidase levels) also appeared normal. Brain MRI showed T2 hyperintensities in genu and splenium of the corpus callosum, the posterior limb of the internal capsule, inferior cerebellar peduncles, particularly decussatio of the medial lemniscus, intraparenchymal and mesencephalic of the trigeminal nerves. The spinal cord was affected over undivided length. Moreover, the lateral corticospinal tracts, posterior columns and anterior spinocerebellar tracts exhibited abnormal signal changes (Fig. 1). Magnetic resonance spectroscopy (MRS) performed on the lesions of the cerebral white matter indicated increased lactate peak. Electroneuromyography (ENMG) was performed and showed no abnormality. Based on the clinical features and the imaging studies, the diagnosis of LBSL was strongly suspected. Hence, DARS2 gene mutation analysis was performed and revealed a "c455G>T (p.C152F) (p.Cys152Phe)/c.22821_228-20delTTinsC" compound heterozygous mutation. The case report includes informed consent for the participation.

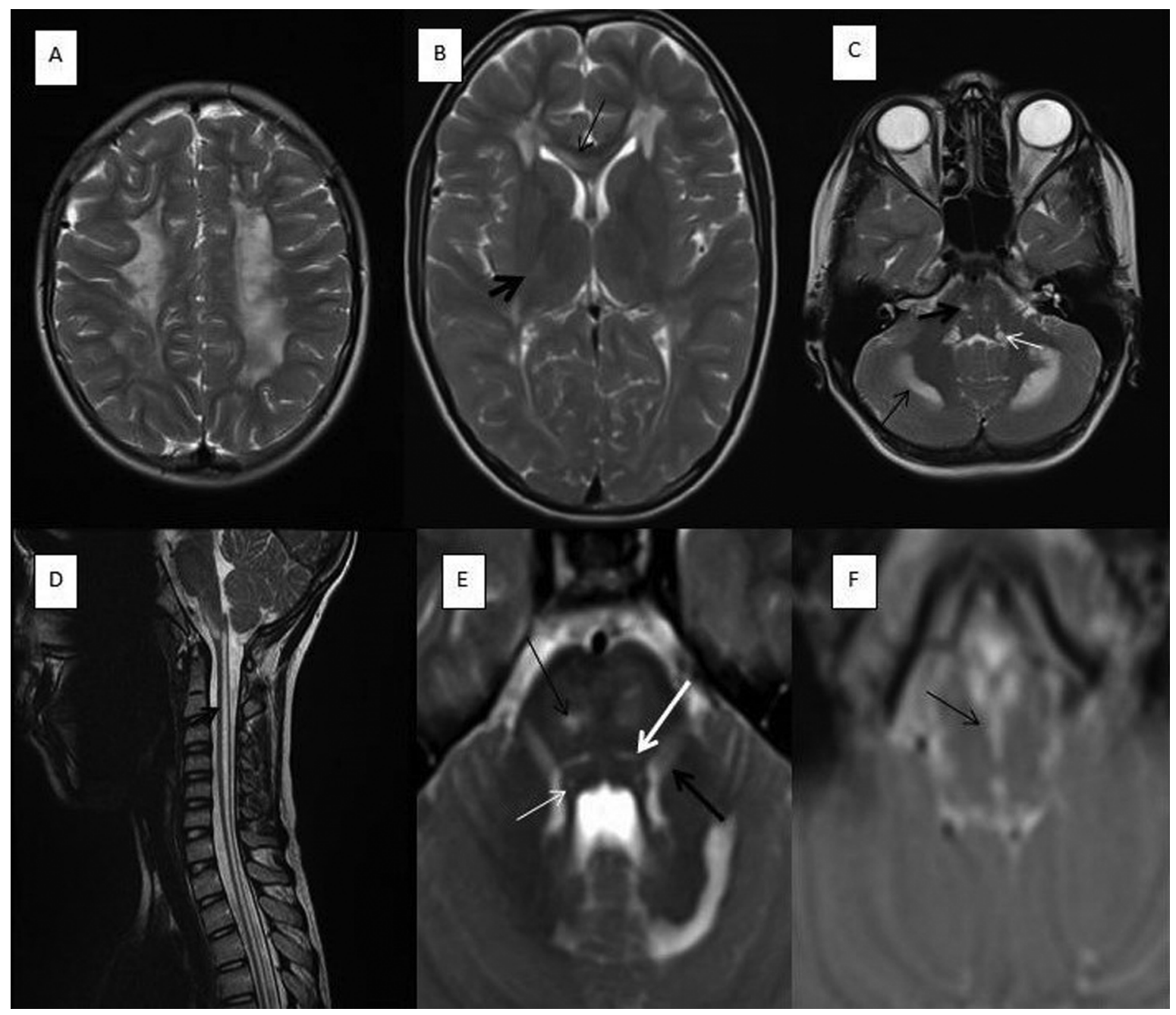

Fig. 1. MRI findings in our patient. (A) Axial T2-weighted image reveals diffuse, patchy and confluent hyperintense lesions in the deep cerebral white matter. (B) The corpus callosum (thin arrow) and posterior part of the internal capsule (thick arrow) show abnormal high signal. (C) There are signal abnormalities in the inferior cerebellar peduncles (white arrow), pyramidal tracts (thick black arrow) and cerebellar subcortical white matter (thin black arrow). (D) The spinal cord displays high signal abnormalities along its whole length. (E) Pyramidal tractus (thin black arrow), medial lemniscus (thick white arrow), mesencephalic trigeminal tractus (thin white arrow) and intraparenchymal part of the trigeminal nerve (thick black arrow) were affected. (F) Decussatio of the medial lemniscus (black arrow) also indicate abnormal high signal. 
Table I. MRI criteria for LBSL. ${ }^{7}$

Signal abnormalities

Major criteria

Cerebral white matter (relative sparing of the subcortical white matter)

Dorsal columns and lateral corticospinal tracts of the spinal cord

Pyramids at the level of the medulla oblongata or decussatio of the medial lemniscus or both

Minor criteria

Splenium of the corpus callosum

Posterior limb of the internal capsule

Superior and inferior cerebellar peduncles

Intraparenchymal part of the trigeminal nerve

Mesencephalic trigeminal tracts

Anterior spinocerebellar tracts in the medulla oblongata

Cerebellar white matter

\section{Discussion}

LBSL is a dissimilar mitochondrial disorder, firstly defined by Van der Knaap et al. ${ }^{2}$ with clinical and neuroimaging findings. A reduced catalytic activity of the encoded mtAspRS has been displayed in some missense mutations. However, no abnormalities in mitochondrial function could be discovered in lymphoblasts, fibroblasts or muscle tissue. ${ }^{3}$ LBSL is generally caused by compound heterozygous for two DARS2 mutations. Previous site-directed mutagenesis studies in yeast have pointed out that alteration of any of the amino acids that present within the active site's hydrogenbond network or the nearby side chains is noxious to the enzyme and any alteration in the C-terminal lobe of the protein leads to a minimum 3-fold decline of enzyme activity about pathogenesis of DARS mutations. In the guidance of these studies, Taft et al. ${ }^{5}$ found a compound-heterozygous mutation in DARS alter amino acids which sits within the active-site pocket. On the basis of similarity in clinical picture and abnormal MRI features of LBSL and hypomyelination with brain stem and spinal cord involvement and leg spasticity (HBSL), caused by compoundheterozygous and homozygous mutations in the DARS gene, it is suggested that the same path also is responsible for pathogenesis of LBSL. ${ }^{5}$ As is known, in general mitochondrial diseases preferably affect the tissues that have a high energy desire. ${ }^{3}$ Therefore, the high expression of mitochondrial tRNAs in the white matter of central nervous system has been estimated as the reason for the selective white matter involvement. ${ }^{6}$ On the other hand, it is indicated that DARS expression, similar to distribution of DARS2, is highly expressed in the ventricular and subventricular zones, including hippocampal subfields, the midlateral temporal cortex, and the frontal polar cortex furthermore DARS immunostaining of the cerebellum, cerebral cortex, hippocampus, and lateral ventricle all show exclusive neuronal staining consistent with clinical presentation. ${ }^{5}$ Moreover, involvement of the organs other than the nervous system has never been reported. Conversely, our patient had undergone unilateral congenital cataract operations. It should prompt to differential diagnosis such as hypomyelination and congenital cataract (HCC) due to the presence of cataract and slowly progressive neurologic impairment. Although bilateral congenital cataract is mostly considered to be a major finding for $\mathrm{HCC}$, the peculiar magnetic resonance pattern of hypomyelination combined with increased periventricular white matter water substance lets distinction of HCC from other diseases even another forms of hypomyelinating leukoencephalopathies. ${ }^{7}$ According to our knowledge, he is the first child diagnosed with LBSL associated with cataract. We may also explain the cause of cataract in our patient with mitochondrial dysfunction.

In the literature, very characteristic evidences in brain and spinal cord MR imaging of LBSL enounce this diagnosis. Conversely, Köhler et al. ${ }^{8}$ reported a patient with a homozygous DARS2 gene mutation with not completing strict MRI signs. However, our patient met the MRI criteria for LBSL including cerebral and cerebellar white matter lesions, together with the involvement of the pyramidal and 
sensory tracts in the brainstem and spinal cord ${ }^{9}$ (Table I ). If we encounter these MRI findings and clinical presentation, another definite diagnosis of HBSL should be considered. It is an uncommon, generally infantile onset leukodystrophy caused by compoundheterozygous and homozygous mutations in cytoplasmic aspartyl-tRNA synthetase (DARS). However, LBSL is caused by mutations in the $D A R S$ mitochondrial paralog, DARS2. It is likewise recommended that attention should be paid to existence of HBSL with the clinical and MRI findings suggesting LBSL, but not carrying DARS2 mutation. ${ }^{1}$ In MRS, lactate is elevated in the affected white matter in LBSL with most of the reported cases in the literature. A few patients with LBSL reported to not show elevated lactate levels in the literature, therefore detectable lactate peaks on MRS are not admitted a mandatory finding2,10. There is no information about the diversity of genotype between with detectable lactate peak and without lactate peaks to our knowledge. Moreover, MRS of our patient showed elevated lactate level in conformity with the literature.

Until now, the extensive majority of LSBL patients had a compound heterozygous mutation in DARS2 gene. Almost all patients with LBSL have a "c.228-21_228-20delTTinsC" mutation at the 3 ' end of the second intron of DARS2 gene. ${ }^{11}$ The "c455G > T (p.C152F) (p.Cys152Phe)/c.228-21_228-20delTTinsC” compound heterozygous mutation was found in our patient. It has also been shown previously in other patients diagnosed with LBSL and is considered as pathogenic mutations. The C152F, Q184K, R263Q and D560V mutations significantly decrease dimerization with the wild-type enzyme. ${ }^{3}$

Looking at the prognosis of LBSL, it was actually defined as a juvenile onset disorder with a slow progression ${ }^{2}$. Van Berge et al. ${ }^{10}$ described that LBSL is a neurological disorder with generally slow progression and low mortality, with in some cases even later onset and slower progress. They also reported that complete wheelchair dependency was uncommon, particularly before the age of 18 years and life expectancy might be normal for most patients. Van Berge et al. ${ }^{10}$ remarked that LBSL had a better prognosis than most other hereditary leukoencephalopathies, but the infantile onset cases characterized by a more rapid neurological deterioration and the most severe cases were concluded with early death. Moreover, we can say that our patient had a slow course, because he was able to walk independently in addition to his examination.

Until now, specific curative treatments are not available for LBSL. Treatment manifestations occur with supportive therapy including physical therapy and rehabilitation to improve motor function for avoiding complications such as contractures and scoliosis. ${ }^{12}$

Finally, LBSL is a rare disease with a slowly progressive course, characterized by predominantly pyramidal, cerebellar and dorsal column dysfunctions. Characteristic MRI findings help to identify patients with LBSL which can be confirmed by the detection of mutations in the DARS2 gene.

\section{REFERENCES}

1. Scheper GC, van der Klok T, van Andel RJ, et al. Mitochondrial aspartyl-tRNA synthetase deficiency causes leukoencephalopathy with brain stem and spinal cord involvement and lactate elevation. Nat Genet 2007; 39: 534-539.

2. Van der Knaap MS, van der Voorn P, Barkhof F, et al. A new leukoencephalopathy with brainstem and spinal cord involvement and high lactate. Ann Neurol 2003; 53: 252-258.

3. Van Berge L, Kevenaar J, Polder E, et al. Pathogenic mutations causing LBSL affect mitochondrial aspartyltRNA synthetase in diverse ways. Biochem J 2013; 450: 345-350.

4. Linnankivi $\mathrm{T}$, Lundbom N, Autti $\mathrm{T}$, et al. Five new cases of a recently described leukoencephalopathy with high brain lactate. Neurology 2004; 63: 688-692.

5. Taft RJ, Vanderver A, Leventer RJ, et al. Mutations in DARS cause hypomyelination with brain stem and spinal cord involvement and leg spasticity. Am J Hum Genet 2013; 92 :774-780.

6. Uluc K, Baskan O, Yildirim KA, et al. Leukoencephalopathy with brain stem and spinal cord involvement and high lactate: a genetically proven case with distinct MRI findings. J Neurol Sci 2008; 273: 118-122.

7. Biancheri R, Zara F, Rossi A, et al. Hypomyelination and congenital cataract: broadening the clinical phenotype. Arch Neurol 2011; 68 :1191-1194.

8. Köhler C, Heyer C, Hoffjan S, et al. Early-onset leukoencephalopathy due to a homozygous missense mutation in the DARS2 gene. Mol Cell Probes 2015; 29: 319-322.

9. Steenweg ME, van Berge L, Van Berkel CG, et al. Earlyonset LBSL: how severe does it get? Neuropediatrics 2012; 43: 332-338. 
10. Van Berge L, Hamilton E.M, Linnankivi T, et al. Leukoencephalopathy with brainstem and spinal cord involvement and lactate elevation: clinical and genetic characterization and target for therapy. Brain 2014; 137: 1019-1029.

11. Van Berge L, Dooves S, van Berkel CG, Polder E, van der Knaap MS, Scheper GC. Leukoencephalopathy with brain stem and spinal cord involvement and lactate elevation is associated with cell-type-dependent splicing of mtAspRS mRNA. Biochem J 2012; 441: 955-962
12. Van der Knaap MS, Scheper GC. Leukoencephalopathy with brain stem and spinal cord involvement and lactate elevation. In: Pagon RA, Bird TC, Dolan CR, et al GeneReviews [Internet]. Seattle (WA): University of Washington, Seattle, 2010: 1993-2017. 